\title{
Article
}

\section{Statistical complexity and two van der Waals' phase transitions}

\author{
F. Penninii ${ }^{1,2}$, A. Plastino ${ }^{3}$
}

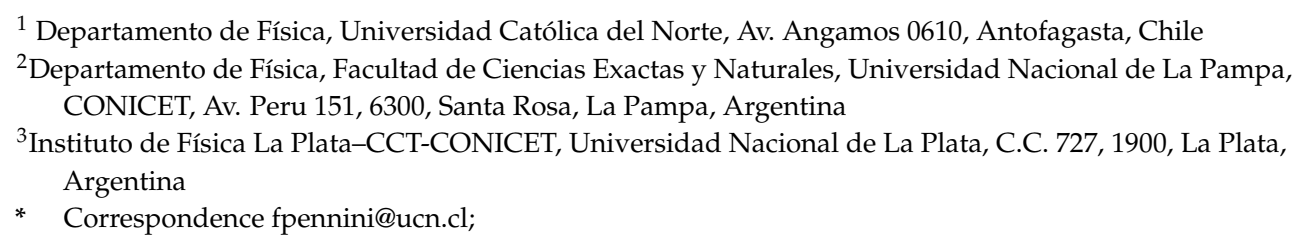

\begin{abstract}
We show that the van der Waals gas does not exhibit just the celebrated liquid-gas (LG) transition but also a quantum-classical (QC) one. We study the issue with reference to the LMC statistical complexity and in relation to the order-disorder contrast (ODC). We show that the ODC is much larger for the QC changeover than for the LG one.
\end{abstract}

Keywords: Statistical complexity, Order-disorder contrast, Phase transitions, van der Waals gas.

\section{Introduction and goal}

The van der Waals (vdW) fluid represents the first and simplest example of a system whose component particles are interacting ones and, most importantly, displays a phase transition (gas-liquid) [1-9]. Here we highlight the fact that there exist a second vdW phase transition (PT) that one immediately encounters by looking at the vdW system in an appropriate light. Thus, we face two differennt vdW phase transitions and we look here at their interplay with regards to the so called statistical complexity $C$ and to the concepts of order-disorder, represented by a quantity called $D$ (both to be defined below).

As just stated, we use the two statistical quantifiers $C$ and $D$ in scrutinizing our two PTs. It will be seen that $C$ and $D$ behave in a marked different fashion with regards to these PTs, that are seen to be quite different from the view point of the statistical quantifiers.

\subsection{Statistical complexity, order, and disorder}

Statistically, maximum disorder is represented by a uniform distribution (UF) [10]. As a consequence, the more different our current probability distribution (CPD) is from the UF, the more order this CPD represents [10]. The associated distance in probability space between CPD and UF is called the disequilibrium $D$ [10]. The product of $D$ with the entropy $S$

$$
C=D S,
$$

is called the statistical complexity. This $C$-expression is the standard complexity form employed in many publications. See, for instance, Refs. [11-18].

\subsection{Organization}

This paper has the following organization. First, in Section 2, we introduce preliminary concepts necessary to discuss our present issues. In Section 3, the ideal gas description is extended to dvW gases. Section 4 incorporates the mean-field picture for a vdW gas. Section 5 explains the quantum-classical transition for the ideal gas while Section 6 is devoted to explore the quantum-classical transition in the others two scenarios considered in this paper, that constitute the central topic of our efforts. In Section 7 we present numerical results and discuss some particular issues. Finally, we draw some conclusions in

Publisher's Note: MDPI stays neutral with regard to jurisdictional claims in published maps and institutional affiliations.

Received:

Accepted:

Published: Section 8. 


\section{Preliminaries}

\subsection{Ideal gas}

The model consists of $N$ mono-atomic identical particles confined to a space of volume $V$ and in thermal equilibrium at temperature $T$, whose Hamiltonian is $\mathcal{H}_{0}=\sum_{i=1}^{N} p_{i}^{2} / 2 m$, with $m$ the mass of the particles and $p_{i}$ the related momenta, $i=1, \ldots, N$. The well-known canonical partition function is [1]

$$
Q_{N}^{(0)}(V, T)=\int \mathrm{d} \Omega \exp \left(-\beta \mathcal{H}_{0}\right)=\frac{1}{N !}\left(\frac{V}{\lambda^{3}}\right)^{N},
$$

with $\mathrm{d} \Omega=\mathrm{d}^{3 N} r \mathrm{~d}^{3 N} p /\left(N ! h^{3 N}\right), \beta=1 / k_{B} T$ ( $k_{B}$ the Boltzmann constant), and $\lambda$ the particles' mean thermal wavelength which is

$$
\lambda=\left(\frac{2 \pi \hbar^{2}}{m k_{B} T}\right)^{1 / 2}
$$

The Helmholtz free energy, obtained after having used the Stirling's approximation $(\ln N ! \approx$ $N \ln N-N)$, becomes

$$
F_{i d e a l}=-N k_{B} T\left[\ln \left(\frac{v}{\lambda^{3}}\right)+1\right]
$$

where $v=V / N$ is the molar volume [1]. Once the free energy is calculated one obtains the rest of the thermodynamic quantities, that we list here for pedagogical purposes. Accordingly, the chemical potential for the ideal gas is [1]

$$
\mu_{\text {ideal }}=k_{B} T \ln \left(\frac{\lambda^{3}}{v}\right) .
$$

Also, the classical entropy provided by the celebrated Sackur-Tetrode equation, is of the form [1]

$$
S_{i d e a l}=-N k_{B} \ln \left(\frac{\lambda^{3}}{v}\right)+\frac{5}{2} N k_{B}=N k_{B} \ln \left[e^{5 / 2}\left(\frac{v}{\lambda^{3}}\right)\right],
$$

which is positive definite if we fulfill the requirement $\lambda^{3} / v \ll e^{5 / 2}$ [20].

Finally, the internal energy is [1]

$$
U_{i d e a l}=F_{i d e a l}+T S_{i d e a l}=\frac{3}{2} N k_{B} T .
$$

\subsection{Statistical quantifiers for the ideal gas}

Now, for a continuous probability distribution, that in this case is the Boltzmann-Gibbs distribution $\rho_{\text {ideal }}(r, p)=\exp \left(-\beta \mathcal{H}_{0}\right) / Q_{N}^{(0)}$, the disequilibrium $D$ reads [10]

$$
D_{\text {ideal }}^{(N)}=\int \mathrm{d} \Omega \rho_{\text {ideal }}^{2}(r, p) .
$$

$D$ can also be obtained from the free energy as shown in Ref. [12]. One obtains

$$
D_{\text {ideal }}^{(N)}=\left(\frac{\lambda^{3}}{v}\right)^{N} e^{-N} 2^{-3 N / 2} .
$$

Now, inserting Eq. (2.6) into the definition (2.8), we obtain the statistical complexity, which is given by [20]

$$
C_{\text {ideal }}^{(N)}=\left(\frac{\lambda^{3}}{v}\right)^{N} e^{-N} 2^{-3 N / 2} \ln \left[e^{5 N / 2}\left(\frac{\lambda^{3}}{v}\right)^{-N}\right]
$$


Remark that if $N \rightarrow \infty$ and/or $T \rightarrow \infty$, the two statistical quantities $D$ and $C$ are zero [19, 20].

In this work we use disequilibrium and complexity per particle, that we name

$$
D_{i d e a l}=\left(\frac{\lambda^{3}}{v}\right) e^{-1} 2^{-3 / 2}
$$

and

$$
C_{i d e a l}=\left(\frac{\lambda^{3}}{v}\right) e^{-1} 2^{-3 / 2} \ln \left[e^{5 / 2}\left(\frac{\lambda^{3}}{v}\right)^{-1}\right]
$$

\section{The van der Waals gas scenario}

The main new ingredient in this case is that of intermolecular interactions in a gas of $N$ identical particles, contained in a volume $V$. One assumes equilibrium at the temperature $T$. In principle, one faces the Hamiltonian [1]

$$
\mathcal{H}=\mathcal{H}_{0}+\sum_{i<j} u_{i j}
$$

Here, $u_{i j}=u\left(\left|r_{i}-r_{j}\right|\right)$ represents the interaction-energy between particles $i$ and $j$, that only depends upon the inter-particle distance $r_{i j}=r_{j}-r_{i}$. The $i, j$ sum runs over the $N(N-1) / 2$ possible particle-pairs [1].

The pertinent canonical partition function $(\mathrm{CPF})$ is [1]

$$
Q_{N}(V, T)=Q_{N}^{(0)}(V, T) Z_{N}(V, T)
$$

$Q_{N}^{(0)}(V, T)$ CFT is that of Eq. (2.2). $Z_{N}(V, T)$ is called the configuration integral $[1,2]$

$$
Z_{N}(V, T)=\frac{1}{V^{N}} \int \mathrm{d}^{3 N_{r}} \exp \left(-\beta \sum_{i<j} u_{i j}\right) .
$$

If $n=N / V \ll 1$ the configuration integral can be approximated by [2]

$$
Z_{N}(V, T) \approx \exp \left(-\frac{N B_{2}(T)}{v}\right)
$$

where $B_{2}(T)$ is the celebrated second virial coefficient [2]. Approximating the interaction as a quantity $u(r)$ as explained below in Eq. (3.20) [2], one has

$$
B_{2}(T)=\frac{1}{2} \int \mathrm{d}^{3} r(1-\exp (-\beta u(r)))
$$

In addition, the grand partition function of the system is given by [1]

$$
Z_{G C}=\sum_{N=0}^{\infty} z^{N} Q_{N}(V, T)
$$

with $z=\exp \left(\mu_{d v W} / k_{B} T\right)$ the fugacity and $\mu_{d v W}$ the chemical potential of the system [1]. Using Eq. (3.14) we analytically get

$$
Z_{G C}=\exp \left[\frac{z V}{\lambda^{3}} \exp \left(-\frac{B_{2}(T)}{v}\right)\right] .
$$

Finally, let us assume that the particle-particle interaction can be simplified in the fashion

$$
u(r)= \begin{cases}\infty & r<r_{o} \\ \exp (-\beta u(r)) \approx 1-\beta u(r) & r>r_{o}\end{cases}
$$


with $r_{o}$ the minimum possible distance amongst molecules [2]. One is led then, using Eq. ( 3.17), to the van der Waals $B_{2}$ expression

$$
B_{2}(T)=b-\beta a,
$$

with $b=2 \pi r_{0}^{3} / 3$ being linked to the volume of a hard-sphere gas, whose mean potential energy reads

$$
a=2 \pi \int_{r_{0}}^{\infty} \mathrm{d} r r^{2} u(r)
$$

3.1. Listing the van der Waals (vdW) gas thermal quantifiers

We are concerned with thermal quantifiers. The Helmholtz free energy $F$ is, for a dvW gas,

$$
F_{d v W}=-k_{B} T \ln \left[Q_{N}(V, T)\right]
$$

with $Q_{N}(V, T)$ represented by Eq. (3.14). Thus, $F_{d v W}$ reads

$$
F_{d v W}=-k_{B} T \ln \left[\frac{1}{N !}\left(\frac{V}{\lambda^{3}}\right)^{N} \exp \left(-\frac{N B_{2}(T)}{v}\right)\right],
$$

that, appealing to Stirling's approximation and noting that $B_{2}=b-a / k_{B} T$, becomes

$$
F_{d v W}=F_{i d e a l}+\frac{N k_{B} T}{v}\left(b-\frac{a}{k_{B} T}\right),
$$

where $F_{\text {ideal }}$ is that of Eq. (2.4) [1]. Now, following text book-recipes [1], from the relation

$$
N=z\left(\frac{\partial \ln Z_{G C}}{\partial z}\right)_{V, T}
$$

we easily are led to a chemical potential of the form

$$
\mu_{d v W}=k_{B} T \ln \left[\frac{\lambda^{3}}{v} \exp \left(\frac{B_{2}(T)}{v}\right)\right] .
$$

The entropy $S$ is given by

$$
S_{d v W}=-\left(\frac{\partial F_{d v W}}{\partial T}\right)_{V, N}
$$

that using Eq. (3.25) becomes

$$
S_{d v W}=S_{i d e a l}-N k_{B}\left(\frac{b}{v}\right),
$$

where $S_{\text {ideal }}$ is gotten from by Eq. (2.6) [1]. The internal energy $U_{d v W}$ reads then

$$
U_{d v W}=F_{d v W}+T S_{d v W}=U_{i d e a l}-\frac{N a}{v},
$$

where $U_{\text {ideal }}$ is given by Eq. (2.7). For the pressure $P_{d v W}$ one has

$$
P_{d v W}=-\frac{1}{N}\left(\frac{\partial F_{d v W}}{\partial v}\right)_{T, N}{ }^{\prime}
$$

so that using Eq. (3.25) we encounter

$$
P_{d v W}=\frac{k_{B} T}{v}\left(1+\frac{b}{v}\right)-\frac{a}{v^{2}} .
$$


For a dilute gas with $v \gg b$ (low density) one specializes this, in the case of the van der Waals gas, to [1]

$$
P_{d v W}=\frac{k_{B} T}{v-b}-\frac{a}{v^{2}}
$$

3.2. Disequilibrium and Complexity in the van der Waals gas

This is the central subject of the present paper. On the basis of the same considerations contemplated above, with the Boltzmann-Gibbs distribution now given by $\rho_{d v W}(r, p)=$ $\exp (-\beta \mathcal{H}) / Q_{N}$, the disequilibrium $D$ is given by

$$
D_{d v W}^{(N)}=\int \mathrm{d} \Omega \rho_{d v W}^{2}(r, p),
$$

so that one immediately finds the vdW disequilibrium per particle (see Ref. [21])

$$
D_{d v W}=D_{\text {ideal }} v \frac{v-\left(b-2 a / k_{B} T\right)}{\left(v-\left(b-a / k_{B} T\right)\right)^{2}}
$$

with $D_{\text {ideal }}$ given by Eq. (2.11).

Therefore, the vdW complexity per particle is

$$
C_{d v W}=D_{d v W}\left(S_{d v W} / k_{B}\right)
$$

that we will compute numerically below.

\section{Mean-field picture for a vdW gas}

This is another subject of interest. A mean field vdW approach. In Ref. [22], its authors describe a mean field picture of the vdW gas. Any given molecule modes in the field generated by all the other molecules, and is regarded otherwise as independent. Thus, if the molecular partition function is called $q$, the total partition function is [22]

$$
Z_{m f}=\frac{q^{N}}{N !}
$$

with

$$
q=\left(\frac{V-N b}{\lambda^{3}}\right) \exp \left(\frac{a}{v k_{B} T}\right)
$$

Accordingly, the Helmholtz free energy is

$$
F_{m f}=-k_{B} T \ln q
$$

i.e.,

$$
F_{m f}=-N k_{B} T\left[1+\ln \left(\frac{v-b}{\lambda^{3}}\right)\right]-\frac{N a}{v},
$$

which coincides with the second virial approximation given in Eq. (27) of Ref. [6]. Further processing easily yields

$$
F_{m f}=-N k_{B} T\left[1+\ln \left(\frac{v-b}{v}\right)+\ln \left(\frac{v-b}{\lambda^{3}}\right)\right]-\frac{N a}{v}
$$

and finally [6]

$$
F_{m f}=F_{i d e a l}-N k_{B} T \ln \left(1-\frac{b}{v}\right)-\frac{N a}{v},
$$

with $F_{\text {ideal }}$ given by Eq. (2.4).

The chemical potential derives from the grand canonical partition function, that in this case reads 


$$
Z_{G C}=\exp \left[z \frac{(V-N b)}{\lambda^{3}} \exp \left(\frac{a}{v k_{B} T}\right)\right] .
$$

Thus, from Eqs. (4.43) and (3.26), we arrive at the chemical potential

$$
\mu_{m f}=k_{B} T \ln \left[\frac{\lambda^{3}}{v} \exp \left(-\frac{2 a}{k_{B} T v}\right)\right] .
$$

Further, the entropy is

$$
S_{m f}=S_{i d e a l}+N k_{B} \ln \left(1-\frac{b}{v}\right),
$$

while the internal energy is obtained using Eqs. (4.42) and (4.45) as [6]

$$
U_{m f}=F_{m f}+T S_{m f}=U_{i d e a l}-\frac{N a}{v},
$$

where $S_{\text {ideal }}$ is gotten from by Eq. (2.6) [1]. The pressure is calculated from

$$
P_{m f}=-\frac{1}{N}\left(\frac{\partial F_{m f}}{\partial v}\right)_{T, N},
$$

which, in view of Eq. (4.42), leads to the van der Waals equation of state given by Eq. (3.32), so that $P_{d v W}=P_{m f}$.

\subsection{Mean field disequilibrium and complexity}

In this case, the disequilibrium is obtained from [12]

$$
D_{m f}=\exp \left[2 \beta\left(F_{m f}(T)-F_{m f}(T / 2) N\right) / N\right],
$$

so that

$$
D_{m f}=D_{\text {ideal }}\left(1-\frac{b}{v}\right)^{-1},
$$

which is independent of $a$. Then, the complexity per particle is

$$
C_{m f}=D_{m f}\left(S_{m f} / k_{B}\right)
$$

\section{Quantum-classical transition in an ideal gas}

\subsection{Generalities}

In this work, the quantum-classical (QC) transition is a central topic. We begin here introducing a brief recompilation of results of Ref. [1], that considers an ideal gaseous system of $N$ non-interacting, indistinguishable particles confined to a space of large volume $V$ and sharing a given energy $E$ [1]. From a quantal view-point we know that the singleparticle energy levels in this system are very close to one another and the energy spectrum is divided into a large number of groups of levels [1]. The average energy of a level is denoted by $\varepsilon$. Thus, if $\mu$ is the chemical potential of the system, the classical limit is attained when [1]

$$
e^{\beta(\varepsilon-\mu)} \gg 1,
$$

or also,

$$
e^{\beta \mu} \ll e^{\beta \varepsilon}
$$

Considering that the mean kinetic energy of a gas molecule in a classical gas is $\varepsilon=$ $U_{i d e a l} / N=3 k_{B} T / 2$, the classical limit is obtained when [20] 


$$
e^{\beta \mu} \ll e^{3 / 2}
$$

\subsection{Ideal gas}

From Eqs. (2.5) and (5.53), we note that the classical regime is attained when

$$
\frac{\lambda^{3}}{v} \ll e^{3 / 2},
$$

or equivalently, taking into account the definition of the mean thermal wavelength (2.3), we have that the gas is classical for temperatures $T$ verifying

$$
T \gg T_{1}=\frac{2 \pi \hbar^{2}}{m k_{B} e v^{2 / 3}}
$$

where $T_{1}$ is the quantum-classical transition-temperature of the ideal gas [20]. At temperature $T_{1}$, the ideal complexity attains its maximum value at $(1 / e)(e / 2)^{3 / 2}=0.582911$. Also, the disequilibrium attains the maximum value at 0.582911 , and the maximum of entropy $S_{\text {ideal }} / k_{B}$ at $1[20]$.

\section{Quantum-classical transition in the van der Waals gas}

This is a transition at which the proper formalism for dealing with de vdW gas changes from classical to quantum. Thus, the transition is quite different from the liquid-gas one, that is to be dealt with with the classical formalism only.

\subsection{Preliminaries}

We extend now our preceding considerations to the vdW gas. To repeat: our aim now is to obtain the classical-quantum transition temperature for $\mathrm{dvW}$ gases. To achieve such an end, we follow the route traced in the above section, taking $\varepsilon=U_{d v W} / N$ equal to the energy per particle of the $\mathrm{dvW}$ gas. Also, we notice that $\mu=\mu_{d v W}$ as the chemical potential of the system. Thus, the new classical regime is now attained at

$$
\exp \left(\beta \mu_{d v W}\right) \ll \exp \left(\beta U_{d v W}\right) .
$$

Considering Eqs. (3.27) and (5.53), the classical regime is attained whenever

$$
\left(\frac{\lambda^{3}}{v}\right) \exp \left(\frac{B_{2}(T)}{v}\right) \ll \exp \left(\beta U_{d v W}\right)
$$

implying that

$$
\frac{\lambda^{3}}{v} \ll \exp \left(\frac{3}{2}-\frac{b}{v}\right)
$$

so that, calling $T_{2}$ the classical-quantum transition ( $\mathrm{dvW}$ gas) temperature, classical instances are attained for temperatures $T$ that verify

$$
T \gg T_{2}=\frac{2 \pi \hbar^{2}}{m k_{B} e v^{2 / 3}} \exp \left(\frac{2 b}{3 v}\right) .
$$

As in the ideal case, the maximum values are $C_{d v W}^{m a x}=D_{d v W}^{m a x}=0.582911$ and, the maximum of entropy $S_{d v W}^{\max } / k_{B}=1$.

\subsection{Mean-field QC temperature}

We proceed in the same way as above. We identify $\varepsilon=U_{m f} / N$ and $\mu=\mu_{m f}$ the enter condition (5.52). Therefore, the classical regime is attained when 


$$
\left(\frac{\lambda^{3}}{v-b}\right) \exp \left(-\frac{a}{k_{B} T v}\right) \ll \exp \left(\beta U_{m f}\right)
$$

i.e.,

$$
\frac{\lambda^{3}}{v-b} \ll \exp \left(\frac{3}{2}\right)
$$

Therefore, the classical scenario obtains at temperatures $T$ that relate to the mean field CQ transition $T_{3}$ as

$$
T \gg T_{3}=\frac{2 \pi \hbar^{2}}{m k_{B} e(v-b)^{2 / 3}} .
$$

As in the ideal gas case, the maximum values are $C_{m f}^{\max }=D_{m f}^{\max }=0.582911$ and, the maximum of entropy $S_{m f}^{\max } / k_{B}=1$.

We conclude that the maxima-values for both $C$ and $D$ coincide in our three scenarios ideal, $\mathrm{vdW}$, and mean field.

\section{Present numerical results}

Entropies per particle (Jaynes' missing information measures [23]) are drawn for Helium in Fig. 1 against temperature in three scenarios:

- $\quad$ vdW gas (called $d v W$ in our graph),

- $\quad$ Idem in the mean field approximation (called $m f$ in the Figure),

- Ideal gas (called ideal in our plot).

Additional relevant data are specified in the Caption. There are four vertical lines. The one at the extreme left corresponds to the temperature $T_{C}$ of the liquid-gas transition. Going from left to right, the three remaining vertical lines correspond, respectively, to the classical-quantum transition for the ideal, vdW, and mean-field formalisms.

The most important feature is that $S_{m f}<S_{d v W}<S_{\text {ideal }}$. The last inequality is an obvious one, as an ideal gas is lacks more information than an attractive one. The interaction adds information, as does the finite molecular volume. An interesting new result is that the mean field approach exaggerates the acquired information (first inequality). It is clear that a mean field provides more "order" than a particle-particle interaction's scenario.

Note that the three curves tend to "meet" at $T_{C}$, the liquid-gas transition temperature. Also that, for helium, $T_{c}<T_{\text {quantum-class. }}$. This happens only in the He-case, not for the remaining noble gases. For He, the gas becomes quantum before becoming a liquid. This is our first novelty of this Section.

Fig. 2 illustrates the behavior of the Disequilibrium $D$ with the temperature $T . D$ is a straightforward measure of the degree of order. The higher $D$ the larger the degree of order. The three scenarios of Fig. 1 are here contemplated as well. We depict not only He-data (left) but also Neon-one (right). The comments made in Fig. 1 with regards to missing information apply here to order-degree, as $D_{i d e a l}<D_{d v W}<D_{m f}$. Note that order-degree suffers a dramatic growth at the QC transition but is unaffected by the liquid-gas one (see Neon instance). In He things are different because before reaching down to $T_{\mathcal{C}}$ we are already at the quantum level. Such is our second novelty here.

Fig. 3 depicts $D$ versus the molecular size. Obviously, order grows with this size (as room for molecules to move diminishes), but more for the $\mathrm{mf}$ treatment than for the one called $\mathrm{dvW}$, as expected after the comments made in this respect above, for Fig. 1. Fig. 4 , instead, represents the statistical complexity versus $T$ in the three instances referred to in Figs. 1 and 2. This is done for He. Note that maximum complexity accrues to the 
quantum-classical transition temperatures, bur not to the liquid-gas ones. This is our third important result of this Section.

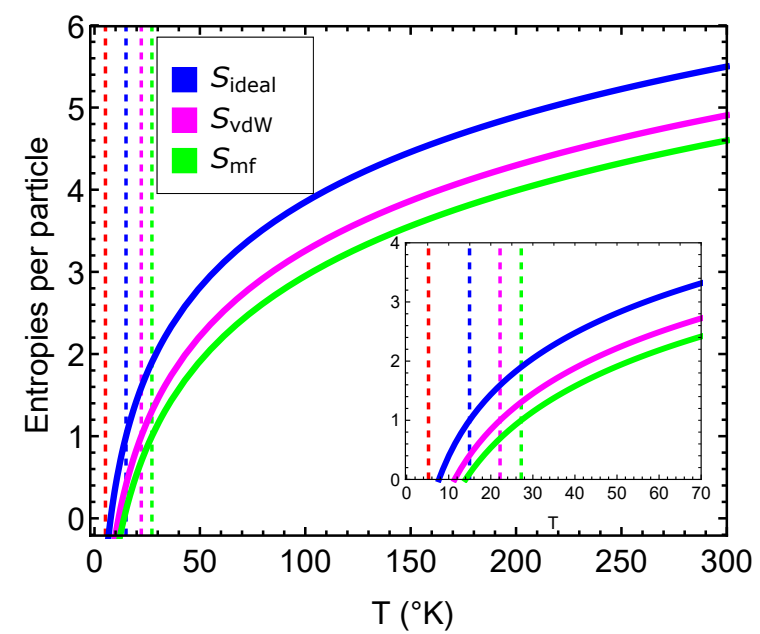

Figure 1. Entropies per particle $S_{d v W} / k_{B}, S_{\text {ideal }} / k_{B}$, and $S_{m f} / k_{B}$, as a function of temperature $T$. We consider the helium molecule, with $a=0.05956 \times 10^{-27}$ ev liters $/ \mathrm{mol}, b=39.418 \times 10^{-27}$ liters $/ \mathrm{mol}$, and mass $m=6.6464731 \times 10^{-27} \mathrm{~kg}$. We take the molar volume $v=0.04$ liters $/ \mathrm{mol}$. The classical-quantum transitions occur at temperature: $T_{1}=14.95^{\circ} \mathrm{K}$ (blue color), $T_{2}=22.204^{\circ} \mathrm{K}$ (magenta color), and $T_{3}=27.24^{\circ} \mathrm{K}$ (green color). The critical gas-liquid temperature is $T_{\mathcal{C}}=5.19^{\circ} \mathrm{K}$ (red color). We appreciate the existence of four vertical lines. The one at the extreme left corresponds to the liquid-gas transition temperature. The three remaining ones to the classical-quantum transition ones, for one for each of our three treatments. Inset: low-temperature enlargement. 


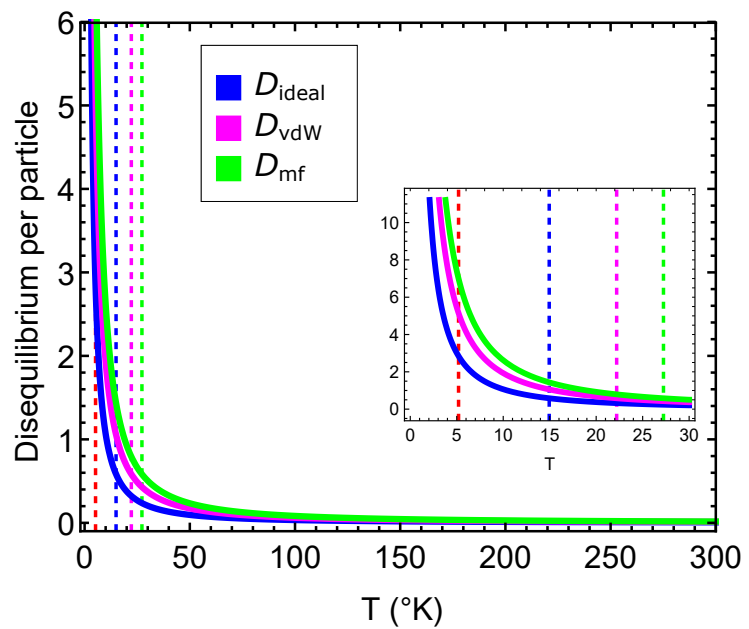

Figure 2. Disequilibrium per particle $D_{d v W}, D_{\text {ideal }}$, and $D_{m f}$, as a function of temperature $T$. We consider the helium molecule, with $a=0.05956 \times 10^{-27} \mathrm{ev}$ liters $/ \mathrm{mol}, b=39.418 \times 10^{-27}$ liters $/ \mathrm{mol}$, and mass $m=6.6464731 \times 10^{-27} \mathrm{~kg}$. We take the molar volume $v=0.04$ liters $/ \mathrm{mol}$. The classicalquantum transitions occur at $T_{1}=14.95^{\circ} \mathrm{K}$ (blue color), $T_{2}=22.204^{\circ} \mathrm{K}$ (magenta color), and $T_{3}=27.24^{\circ} \mathrm{K}$ (green color). The critical temperature is $T_{c}=5.19^{\circ} \mathrm{K}$ (red color). Inset: low-temperature enlargement.

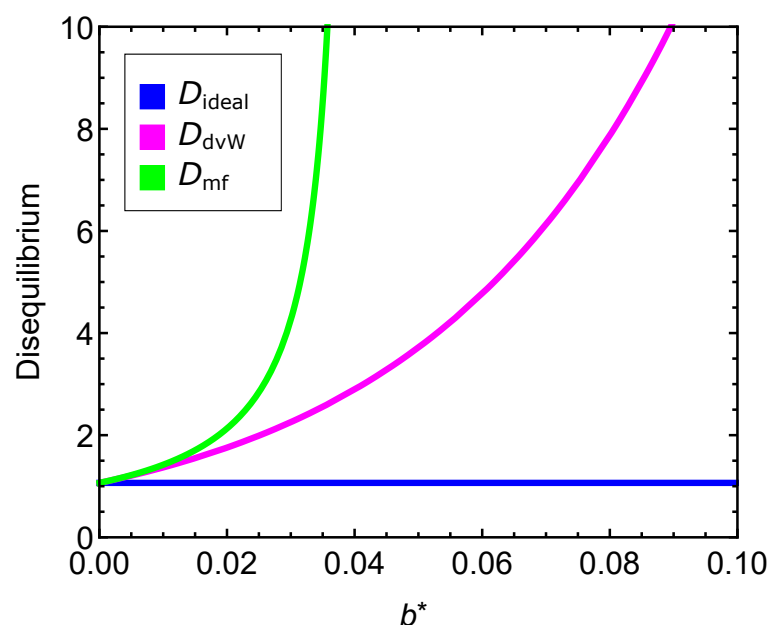

Figure 3. Disequilibrium per particle $D_{d v W}, D_{i d e a l}$, and $D_{m f}$ as a function of the parameter $b^{*}=$ $N_{A} b$, with $N_{A}=6.022140 \times 10^{23}$ the Avogadro constant. We take the molar volume $v=0.04$ liters $/ \mathrm{mol}$, and temperature $T=10^{\circ} \mathrm{K}$. We consider $a=0.05956 \times 10^{-27} \mathrm{ev} \mathrm{liters} / \mathrm{mol}$, and a mass $m=6.6464731 \times 10^{-27} \mathrm{~kg}$ (Helium data with free $b$ coefficient). 


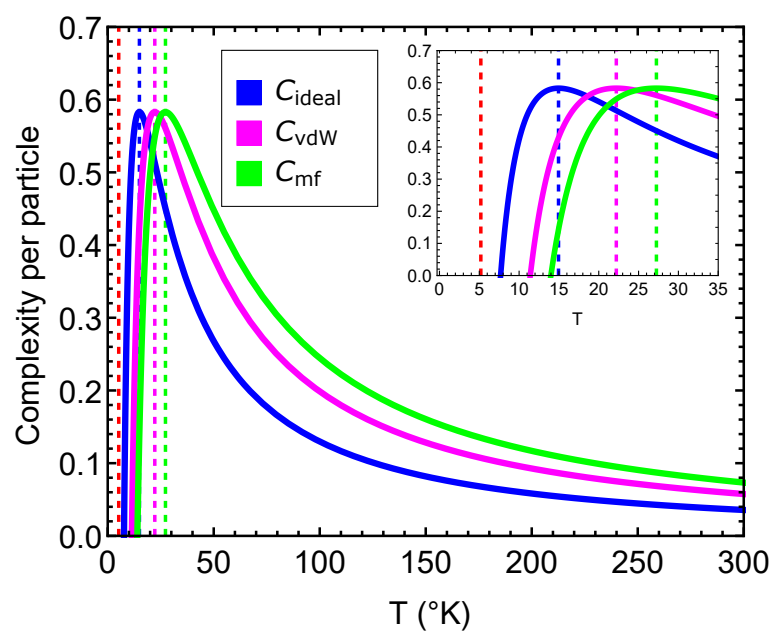

Figure 4. Complexity quantifier versus temperature. Most of the details to be described are those of Fig. 1. Accordingly, we plot complexities per particle $C_{d v W}, C_{i d e a l}$, and $C_{m f}$, as a function of temperature $T$. We consider the helium molecule, with $a=0.05956 \times 10^{-27} \mathrm{ev} \mathrm{liters} / \mathrm{mol}$, $b=39.418 \times 10^{-27}$ liters $/ \mathrm{mol}$, and the mass $m=6.6464731 \times 10^{-27} \mathrm{~kg}$. We take the molar volume $v=0.04$ liters $/ \mathrm{mol}$. The classical-quantum transitions occur at temperature: $T_{1}=14.95^{\circ} \mathrm{K}$ (blue color), $T_{2}=22.204^{\circ} \mathrm{K}$ (magenta color), and $T_{3}=27.24^{\circ} \mathrm{K}$ (green color). The critical temperature is $T_{c}=5.19^{\circ} \mathrm{K}$ (red color). Inset: low-temperature enlargement.

\begin{tabular}{|c|c|c|c|c|}
\hline Gas name & $T_{1}\left({ }^{\circ} \mathrm{K}\right)$ & $T_{2}\left({ }^{\circ} \mathrm{K}\right)$ & $T_{3}\left({ }^{\circ} \mathrm{K}\right)$ & $T_{\mathcal{C}}\left({ }^{\circ} \mathrm{K}\right)$ \\
\hline Helium & 11.4082 & 14.8513 & 15.9594 & 5.19 \\
Neon & 2.26279 & 2.74122 & 2.83708 & 44.49 \\
Argon & 1.14304 & 1.63484 & 1.90927 & 150.70 \\
Krypton & 0.54491 & 0.844234 & 1.11144 & 209.52 \\
Xenon & 0.34779 & 0.616651 & 1.28417 & 289.73 \\
\hline
\end{tabular}

Table 1: Classical-quantum transition temperature for Noble gases (three left columns). The molar volume considered in this table is $v=0.06$ liters $/ \mathrm{mol}$. Also, the temperature $T_{\mathcal{C}}$ is listed in the fourth column.

Table 1 lists, from left to right, the three temperatures corresponding to the quantumclassical transition $T_{i}, i=1,2,3$, corresponding to our three treatments explicitated with reference to Fig. 1. The fourth column is reserved to the liquid-gas transition temperature $T_{c}$. This is done for five noble gases. 


\section{Conclusions}

We have studied here the interplay between order and disorder for the van der Waals gas, with focus in low temperatures $T$. The interrelationship between order and disorder is faithfully represented by the statistical quantifier called disequilibrium $D$. The essential fact is that at low $T$ there exist not one but two phase transitions. In addition to the celebrated (I) gas-liquid one there emerges a (II) classical-quantum one.

We have examined details of the QV transition temperatures for the van der Waals gas and for its mean field approximation. For comparison's sake, the ideal gas instance is also examined in this light.

We conclude that the maxima-values for both $C$ and $D$ coincide in our three scenarios ideal, $\mathrm{vdW}$, and mean field.

The classical-quantum Transition produces dramatic effects in the order-disorder (represented by $D$ ) transactions, greatly augmenting the order-degree, while the gas-liquid transition generates just a moderate order-growth.

As for the statistical complexity $C$, it exhibits a maximum at the $Q C$ transition II.

We conclude that our two phase transitions are quite different from an statistical view point, for which the quantum-classical $(\mathrm{QC})$ one is of a much more drastic nature that the liquid-gas (LG) phase transition. This should be expected. The QS one entails a change in the formalism to be employed, while the LG transition is here described classically.

Author Contributions Investigation, F.P., and A.P.; Project administration, F.P.; Writingoriginal draft, F.P., and A.P. All authors have read and agreed to the published version of the manuscript.

Funding Research was partially supported by FONDECYT, grant 1181558 and by CONICET (Argentine Agency) Grant PIP0728.

Conflicts of Interest The authors declare no conflict of interest. 


\section{References}

1. Pathria, R.K. Statistical Mechanics, 2nd., Butterworth-Heinemann, Oxford, UK, 1996.

2. Reif, F. Fundamentals of Statistical and Thermal Physics, 1 ed., Waveland Press, 2009.

3. Landsberg, P.T. Problems in Thermodynamics and Statistical Physics, PION, London, 1971.

4. Van der Waals, J.D. Nobel Lecture, December 12, 1910.

5. Kittel C., Kroemer, H. Thermal Physics, 2nd edition, Freeman, New York, 1980.

6. Johnston, D.C. Advances in Thermodynamics of the van der Waals Fluid, Morgan and Claypool Publishers, San Rafael, California, 2014.

7. De Boer, J. Van der Waals in his time and the present revival. Physica 73 (1974) 1-27.

8. Sadus, R.J. The Dieterici alternative to the van der Waals approach for equations of state: second virial coefficients. Phys. Chem Chem Phys 4, 2002, 919-921.

9. MacDougall, F.H. The equation of state for gases and liquidas. J. Am. Chem. Soc. 38, 1916, 528-555.

10. López-Ruiz, R., Mancini, H.L., Calbet, X. A statistical measure of complexity. Phys. Lett. A 209, 1995, 321-326.

11. López-Ruiz, R. Complexity in some physical systems. International Journal of Bifurcation and Chaos 11, 2001, 2669-2673.

12. Martin, M.T., Plastino, A., Rosso, O.A., Statistical complexity and disequilibrium. Phys. Lett. A 311 (2003) 126-132.

13. Rudnicki, L., Toranzo, I.V., Sánchez-Moreno, P., Dehesa, J.S. Monotone measures of statistical complexity. Phys. Lett. A 380, 2016, 377-380.

14. López-Ruiz, R. A Statistical Measure of Complexity in Concepts and recent advances in generalized infpormation measures and statistics, A. Kowalski, R. Rossignoli, E.M.C. Curado (Eds.), Bentham Science Books, pp. 147-168, New York, 2013.

15. Sen, K.D. (Editor), Statistical Complexity. Applications in elctronic structure, Springer, Berlin 2011.

16. Martin, M.T., Plastino, A., Rosso, O.A. Generalized statistical complexity measures: Geometrical and analytical properties. Physica A, 369, 2006, 439-462.

17. Anteneodo, C., Plastino, A.R. Some features of the López-Ruiz-Mancini-Calbet (LMC) statistical measure of complexity. Phys. Lett. A 223, 1996, 348-354.

18. Piasecki, R., Plastino, A. Entropic descriptor of a complex behaviour. Physica A 389, 2010, 397-407.

19. Pennini, F., Plastino, A. Statistical complexity, virial expansion, and van der Waals equation. Physica A 458, 2016, 239-247.

20. Branada, R., Pennini, F., Plastino, A. Statistical complexity and classical-quantum frontier, Physica A 511, 2018, 18-26.

21. Pennini, F., Plastino, A., Yañez, J., Ferri, G.L. Free energies divergences as statistical quantifiers. Physica A 564, 2021, 125505.

22. Clark, A., de Llano, M. A van der Waals Theory of the crystalline state. American Journal of Physics 45, 1977, 247-251.

23. Katz, A. Principles of Statistical Mechanics: The Information Theory Approach, Freeman, San Fransisco, 1965. 
